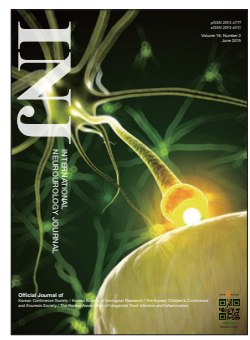

\title{
Alpha-Blocker Treatment Response in Men With Lower Urinary Tract Symptoms Based on Sympathetic Activity: Prospective, Multicenter, Open-Labeled, Observational Study
}

\author{
${ }^{1}$ Department of Urology, Ajou University School of Medicine, Suwon, Korea \\ ${ }^{2}$ Department of Urology and Urological Science Institute, Yonsei University College of Medicine, Seoul, Korea \\ ${ }^{3}$ Department of Urology, Samsung Medical Center, Sungkyunkwan University School of Medicine, Seoul, Korea \\ ${ }^{4}$ Department of Urology, Chonbuk National University Medical School, Jeonju, Korea \\ ${ }^{5}$ Department of Urology, Chonnam National University Medical School, Gwangju, Korea \\ ${ }^{6}$ Department of Urology, Korea University Anam Hospital, Seoul, Korea \\ ${ }^{7}$ Department of Urology, Dongguk University College of Medicine, Gyeongju, Korea \\ ${ }^{8}$ Department of Urology, Dankook University College of Medicine, Cheonan, Korea \\ ${ }^{9}$ Department of Urology, Inha University College of Medicine, Incheon, Korea \\ ${ }^{10}$ Department of Urology, Seoul National University Hospital, Seoul, Korea \\ ${ }^{11}$ Department of Urology, The Catholic University of Korea College of Medicine, Seoul, Korea
}

Sung Gon Park ${ }^{1}$, Byung Ha Chung'2, Sung Won Lee ${ }^{3}$, Jong Kwan Park ${ }^{4}$, Kwangsung Park ${ }^{5}$, Jun Cheon ${ }^{6}$, Kyung Seop Lee Hyung-Jee Kim ${ }^{8}$, Do-Hwan Seong', Seung-June Oh ${ }^{10}$, Sae Woong Kim ${ }^{11}$, Ji Youl Lee ${ }^{11}$, Seol Ho Choo ${ }^{1}$, Jong Bo Choi ${ }^{1}$

Purpose: In this study, we compared the treatment outcomes for an a-blocker between 2 groups of men, one with high sympathetic activity (HSA) and another with low sympathetic activity (LSA) or normal sympathetic activity.

Methods: A total of 159 men ( $\geq 50$ years of age) with lower urinary tract symptoms resulting from benign prostatic hyperplasia were analyzed. We assigned patients to groups according to their sympathetic activity, which was evaluated by heart rate variability measurements. HSA was defined as a low frequency/high frequency ratio greater than 1.6. All patients received 10 mg of alfuzosin once a day for 12 weeks. The primary end point was a change in the total International Prostate Symptom Score (IPSS) at 12 weeks from baseline.

Results: Sixty-seven men were assigned to the HSA group and 92 men were assigned to the LSA group. The baseline characteristics were not significantly different between the 2 groups, and the response to alfuzosin was good in both groups. The mean total IPSS change was not different between the groups. Both groups were not significantly different with respect to the changes in maximal flow rate, IPSS voiding or storage symptom subscores, quality of life, and rates of adverse drug events. The HSA group showed a similar willingness to continue treatment compared to the LSA group, although their treatment satisfaction rating was lower.

Conclusions: The therapeutic effects of alfuzosin did not differ in regards to the differences in sympathetic activity, but treatment satisfaction ratings were lower in the HSA group.

Keywords: Heart Rate; Prostatic Hyperplasia; Sympathetic Nervous System; Lower Urinary Tract Symptoms

- Research Ethics: This study was approved by Institutional Review Board of Ajou University Hospital (IRB No. MED-SUR-10-338).

- Conflict of Interest: No potential conflict of interest relevant to this article was reported.

Corresponding author: Seol Ho Choo (iD http://orcid.org/0000-0003-4357-4330 Department of Urology, Ajou University Hospital, Ajou University School of Medicine,

164 World cup-ro, Yeongtong-gu, Suwon 443-380, Korea

E-mail: predoc@naver.com / Tel: +82-31-219-5270 / Fax: +82-31-219-5276

Co-corresponding author: Ji Youl Lee id http://orcid.org/0000-0001-6775-1157

Department of Urology, Seoul St. Mary's Hospital, The Catholic University of Korea College

of Medicine, 222 Banpo-daero, Seocho-gu, Seoul 137-701, Korea

E-mail: uroljy@catholic.ac.kr / Tel: +82-2-2258-6227 / Fax: +82-2-599-7839

Submitted: March 27, 2015 / Accepted after revision: May 29, 2015

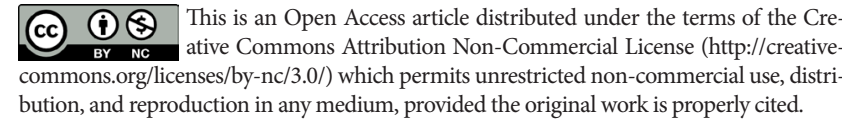

bution, and reproduction in any medium, provided the original work is properly cited. 


\section{INTRODUCTION}

Benign prostate hyperplasia (BPH) is a disease primarily prevalent in elderly men. Many of individuals with BPH have lower urinary tract symptoms (LUTS) that hinder general activity and deteriorate quality of life (QoL) [1,2]. Although transurethral surgery can provide effective relief, medical treatment with careful follow-up is the first-line treatment strategy for BPH [3]. Alpha-blockers relieve bladder outlet obstruction through the smooth muscle relaxation in the prostate, prostatic capsule, base, and trigone of the bladder [3,4], and are an effective and rapid available treatment [5]. Previous studies have argued that LUTS in males is associated with sympathetic nervous system activity. Sympathetic overactivity has been associated with more rapid BPH development [6], while autonomic nervous system (ANS) hyperactivity is also significantly associated with LUTS [7]. Choi et al. [8] have argued that men with LUTS have higher sympathetic activity, and of those individuals, men with voiding symptom-predominant LUTS have relatively higher sympathetic activity than men with storage symptom-predominant LUTS. Since these differences in sympathetic activity are associated with differences in LUTS, $\alpha$-blocker treatment outcomes may also be different, depending on sympathetic activity.

There are many methods that can be used to evaluate autonomic activity, including measuring the Valsalva ratio or performing a tilt-table or thermoregulatory sweat test [9]. The measurement of heart rate variability (HRV) is also a well-established noninvasive assessment tool that can be used to evaluate autonomic nervous activity [10].

In this study, we used HRV measurements to compare treatment outcomes of an a-blocker for LUTS in men with increased sympathetic activity compared to men with normal or lower sympathetic activity.

\section{MATERIALS AND METHODS}

\section{Patients and Study Design}

This prospective, multicenter, open-labeled observational study was conducted in 11 hospitals in Korea. The study protocol was reviewed and approved by The Institutional Review Board of each study center. The enrolled patients suffered from LUTS associated with BPH and were deemed to be fit for a-blocker treatment based on the decision of their physicians. The inclusion criteria included age $\geq 50$ years old, total International Prostate Symptom Score (IPSS) $\geq 8$, maximum urinary flow rate $(\mathrm{Qmax}) \leq 15 \mathrm{~mL} / \mathrm{sec}$, and a period of more than 3 months with LUTS. Subjects with the following conditions were excluded from the study: allergic drug reaction to $a$-blockers, orthostatic hypotension, a history of prostatectomy, renal impairment, severe hepatic disorders, a prostate-specific antigen level higher than $10 \mathrm{ng} / \mathrm{mL}$, the use of any a-blocker in the 4 weeks prior to baseline measurements, or the use of 5 alpha-reductase inhibitors in the 6 months prior to baseline measurements. Patients who were currently receiving or were planning to take the following drugs that could influence ANS were excluded: $\alpha-$ or $\beta$-receptor agonists or antagonists, anticholinergics, antihypertensive drugs, antipsychotics, anxiolytics, or antidepressants.

The primary end point of the study was the change in total IPSS at 12 weeks from baseline. The secondary endpoints included changes in IPSS subscores, QoL, Qmax, and postvoid residual (PVR) volume ratio (residual volume/[voided volume+residual volume]) at 12 weeks, and a benefit, satisfaction with treatment, and willingness to continue treatment questionnaire (BSW) [11] at 12 weeks.

After screening, HRV parameters were measured using an in-house system, according to the standard methods described in previous studies $[8,10]$. Consumption of food, tea, or coffee and cigarette smoking before the HRV measurement were restricted in order to avoid any potential influence on the ANS. After 30 minutes of rest, each patient underwent an electrocardiographic signal recording for 5 minutes while sitting (SA3000P, Medicore, Seoul, Korea). We found that the mean value of the low frequency/high frequency (LF/HF) ratio in healthy elderly men was approximately 1.6 , using data from a pilot study of 118 healthy volunteers (40-70 years of age) who had a total IPSS lower than 8. Therefore, patients with an LF/HF ratio $\geq 1.6$ were assigned to the high sympathetic activity (HSA) group, while those with an LF/HF lower than 1.6 were assigned to the low sympathetic activity (LSA) group.

All patients were given $10 \mathrm{mg}$ of alfuzosin once a day for 12 weeks. The IPSS and QoL scores, Qmax, PVR, overactive bladder symptom score (OAB-SS), and simplified International Index of Erectile Function (IIEF-5) were assessed at 4 and 12 weeks. BSW was obtained at 12 weeks. State anxiety and trait anxiety were evaluated using the Korean version of the Spielberger State Trait Anxiety Inventory [12] at baseline and at 12 weeks. All adverse events and adverse drug reactions were recorded and assessed for severity and causal relationship. 


\section{Statistical Analysis}

The Student t-test and Wilcoxon rank sum test were used to analyze continuous variables, and the chi-square test was used to analyze the categorical variables. For variables that differed at baseline, the analysis of covariance test was performed to compare 2 groups. All results are expressed as the mean \pm standard deviation. All statistical evaluations were performed using IBM SPSS Statistics ver. 19.0 (IBM Co., Armonk, NY, USA). A P-value of less than 0.05 was considered to be significant.

\section{RESULTS}

Between June 2011 and February 2013, 159 patients were assigned to the HSA group $(n=67)$ or to the LSA group $(n=92)$, according

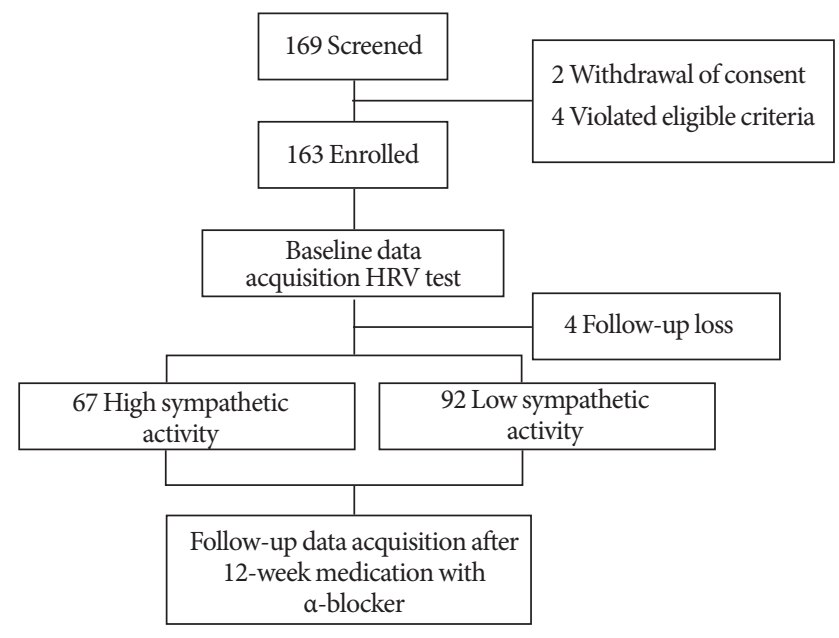

Fig. 1. Patient disposition. HRV, heart rate variability. to their LF/HF ratio (Fig. 1). The baseline characteristics of both groups are presented in Table 1. The HSA and LSA groups did not differ in mean age $(60.0 \pm 5.8$ and $62.5 \pm 7.9$ years, respectively, $\mathrm{P}=0.061)$, mean body mass index $(23.7 \pm 2.5$ and $23.3 \pm 2.6 \mathrm{~kg} /$ $\mathrm{m}^{2}$, respectively, $\left.\mathrm{P}=0.476\right)$, IIEF-5 $(12.9 \pm 6.6$ and $14.1 \pm 7.6$, respectively, $\mathrm{P}=0.219)$ and total IPSS $(16.8 \pm 6.2$ and $16.6 \pm 6.5$, respectively, $\mathrm{P}=0.741)$. However, the HSA and LSA groups differed in QoL (4.0 \pm 0.8 and 3.6 \pm 1.1 , respectively, $\mathrm{P}=0.045)$, Qmax (9.2 \pm 3.6 and $11.3 \pm 4.5 \mathrm{~mL} / \mathrm{sec}$, respectively, $\mathrm{P}=0.001)$, and $\mathrm{PVR}$ $(22.0 \pm 25.1$ and $36.6 \pm 36.7 \mathrm{~mL}$, respectively, $\mathrm{P}=0.007)$ (Table 1). Alfuzosin treatment induced a significant improvement in LUTS for both groups. The total changes for IPSS at 4 weeks were $-3.63 \pm 5.19$ in the HSA group and $-2.92 \pm 6.32$ in the LSA group. The Qmax improvements were 2.34 \pm 5.07 in the HSA group and $2.29 \pm 4.80$ in the LSA group. After 12 weeks of treatment, the mean total IPSS change in the HSA group did not differ from the LSA group $(-3.67 \pm 5.61$ and $-3.88 \pm 6.04$, respectively, $\mathrm{P}=0.817)$. The HSA and LSA groups did not differ on the following parameters: changes in Qmax (3.31 \pm 6.42 and 2.63 \pm 6.39 , respectively, $\mathrm{P}=0.880)$, PVR ratio $(-1.81 \% \pm 8.84 \%$ and $-2.95 \% \pm 12.18 \%$, respectively, $\mathrm{P}=0.912$ ), IPSS voiding symptom subscore $(-2.52 \pm 4.23$ and $-2.48 \pm 4.51$, respectively, $\mathrm{P}=0.964)$, IPSS storage symptom subscore $(-1.15 \pm 2.08$ and $-1.40 \pm 2.33$, respectively, $\mathrm{P}=0.694)$, QoL $(-0.45 \pm 0.97$ and $-0.51 \pm 1.29$, respectively, $\mathrm{P}=0.204)$, OAB-SS $(-0.40 \pm 1.29$ and $-0.17 \pm 1.51$, respectively, $\mathrm{P}=0.549)$, and rates of adverse drug events (13.2\% and 9.7\%, respectively, $\mathrm{P}=0.479)$. The HSA group showed slightly more improvement in changes in IIEF-5 compared to the LSA group $(1.14 \pm 5.34$ and $-0.71 \pm 5.04$, respectively, $\mathrm{P}=0.049)$. The changes in state anxiety (HSA, $-1.42 \pm 5.28$; LSA, $-1.76 \pm 6.64, \mathrm{P}=0.837$ )

Table 1. Baseline characteristics

\begin{tabular}{lccr}
\hline Characteristic & HSA group $(\mathrm{n}=67)$ & LSA group $(\mathrm{n}=92)$ & P-value \\
\hline Age $(\mathrm{yr})$ & $60.0 \pm 5.8$ & $62.5 \pm 7.9$ & 0.061 \\
Body mass index $\left(\mathrm{kg} / \mathrm{m}^{2}\right)$ & $23.7 \pm 2.5$ & $23.3 \pm 2.6$ & 0.476 \\
LF/HF ratio & $3.97 \pm 4.52$ & $0.91 \pm 0.4$ & $<0.001$ \\
IPSS total & $16.8 \pm 6.2$ & $16.6 \pm 6.5$ & 0.741 \\
Voiding subscore & $10.4 \pm 4.3$ & $10.2 \pm 4.9$ & 0.665 \\
Storage subscore & $6.4 \pm 3.1$ & $6.5 \pm 3.1$ & 0.718 \\
Quality of life & $4.0 \pm 0.8$ & $3.6 \pm 1.1$ & 0.045 \\
Qmax (mL/sec) & $9.2 \pm 3.6$ & $11.3 \pm 4.5$ & 0.001 \\
Postvoid residual $(\mathrm{mL})$ & $22.0 \pm 25.1$ & $36.6 \pm 36.7$ & 0.007
\end{tabular}

Values are presented as mean \pm standard deviation.

HSA, high sympathetic activity; LSA, low sympathetic activity; LF/HF, low frequency/high frequency; IPSS, International Prostate Symptom Score; Qmax, maximum urinary flow rate. 
and changes in trait anxiety (HSA, $-0.46 \pm 5.67$; LSA, $-1.73 \pm 5.69$; $\mathrm{P}=0.284$ ) did not differ between the groups (Table 2). With respect to BSW, the HSA group reported that they received a similar benefit (HSA, 74.2\%; LSA, 83.9\%; $\mathrm{P}=0.243$ ) and had a similar willingness to continue treatment (HSA, 76.7\%; LSA, 90.1\%; $\mathrm{P}=0.053)$ compared to the LSA group. However, treatment satisfaction ratings in the HSA group were lower than the LSA group (HSA, 72.1\%; LSA, 87.7\%; P = 0.049).

\section{DISCUSSION}

The dynamic component yields great importance in the pathology of $\mathrm{BPH}$. Although the prostate size is not related to the severity of BPH symptoms [13], urethral obstruction is directly correlated with the amount of prostatic smooth muscle [14]. The prostate is innervated by both the sympathetic and parasympathetic nervous systems [15]. Sympathetic innervation is essential to the regulation of stromal cells and may provide

Table 2. Changes of clinical characteristics from baseline after 12 weeks of treatment

\begin{tabular}{|c|c|c|c|}
\hline Variable & HSA group $(n=67)$ & LSA group $(n=92)$ & P-value \\
\hline \multicolumn{4}{|l|}{ IPSS total } \\
\hline Baseline & $16.8 \pm 6.2$ & $16.6 \pm 6.5$ & 0.741 \\
\hline Change from baseline & $-3.67 \pm 5.61$ & $-3.88 \pm 6.04$ & 0.817 \\
\hline \multicolumn{4}{|l|}{ Voiding subscore } \\
\hline Baseline & $10.4 \pm 4.3$ & $10.2 \pm 4.9$ & 0.665 \\
\hline Change from baseline & $-2.52 \pm 4.23$ & $-2.48 \pm 4.51$ & 0.964 \\
\hline \multicolumn{4}{|l|}{ Storage subscore } \\
\hline Baseline & $6.4 \pm 3.1$ & $6.5 \pm 3.1$ & 0.718 \\
\hline Change from baseline & $-1.15 \pm 2.08$ & $-1.40 \pm 2.33$ & 0.694 \\
\hline \multicolumn{4}{|l|}{ Quality of life } \\
\hline Baseline & $4.0 \pm 0.8$ & $3.6 \pm 1.1$ & 0.045 \\
\hline Change from baseline & $-0.45 \pm 0.97$ & $-0.51 \pm 1.29$ & 0.204 \\
\hline \multicolumn{4}{|l|}{$\mathrm{Qmax}(\mathrm{mL} / \mathrm{sec})$} \\
\hline Baseline & $9.2 \pm 3.6$ & $11.3 \pm 4.5$ & 0.001 \\
\hline Change from baseline & $3.31 \pm 6.42$ & $2.63 \pm 6.39$ & 0.880 \\
\hline \multicolumn{4}{|l|}{ Ratio of PVR (\%) } \\
\hline Baseline & $11.6 \pm 11.7$ & $15.0 \pm 15.1$ & 0.174 \\
\hline Change from baseline & $-1.81 \pm 8.84$ & $-2.95 \pm 12.18$ & 0.912 \\
\hline \multicolumn{4}{|l|}{$\operatorname{PVR}(\mathrm{mL})$} \\
\hline Baseline & $22.0 \pm 25.1$ & $36.6 \pm 36.7$ & 0.007 \\
\hline Change from baseline & $-0.03 \pm 25.24$ & $-9.86 \pm 29.81$ & 0.530 \\
\hline \multicolumn{4}{|l|}{ IIEF-5 } \\
\hline Baseline & $12.9 \pm 6.6$ & $14.1 \pm 7.6$ & 0.219 \\
\hline Change from baseline & $1.14 \pm 5.34$ & $-0.71 \pm 5.04$ & 0.049 \\
\hline \multicolumn{4}{|l|}{ OAB-SS } \\
\hline Baseline & $3.9 \pm 2.4$ & $3.5 \pm 2.1$ & 0.310 \\
\hline Change from baseline & $-0.40 \pm 1.29$ & $-0.17 \pm 1.51$ & 0.549 \\
\hline \multicolumn{4}{|l|}{ STAI - state anxiety } \\
\hline Baseline & $40.8 \pm 10.0$ & $39.5 \pm 10.8$ & 0.447 \\
\hline Change from baseline & $-1.42 \pm 5.28$ & $-1.76 \pm 6.64$ & 0.837 \\
\hline \multicolumn{4}{|l|}{ STAI - trait anxiety } \\
\hline Baseline & $41.1 \pm 9.7$ & $41.3 \pm 11.1$ & 0.903 \\
\hline Change from baseline & $-0.46 \pm 5.67$ & $-1.73 \pm 5.69$ & 0.284 \\
\hline
\end{tabular}

Values are presented as mean \pm standard deviation.

HSA, high sympathetic activity; LSA, low sympathetic activity; IPSS, International Prostate Symptom Score; Qmax, maximum urinary flow rate; PVR, postvoid residual; IIEF-5, International Index of Erectile Function; OAB-SS, overactive bladder symptom score; STAI, state-trait anxiety inventory. 
prostatic muscle tone. Alpha-adrenergic receptors are concentrated in stromal cells and in the prostatic blood vessels [15]. Prostate growth is also associated with autonomic nervous activity. In a study with aging rats, autonomic activity increases facilitated the development of prostatic hyperplasia; removal of this innervation resulted in a regression in prostate gland weight [16]. Many previous studies have suggested an association between elevated sympathetic activity and BPH with LUTS, such that more rapidly developing BPH is associated with sympathetic overactivity [6]. Meigs et al. [17] showed that sympathetic activation, as measured by increased serum markers of heart disease and increased $\beta$-blocker use, is associated with symptomatic BPH. McVary et al. [7] evaluated various markers of ANS activity in men who had BPH with LUTS, and reported that ANS hyperactivity was associated with LUTS severity and prostate size.

There are many methods to evaluate ANS activity, and analysis of HRV is one of them. The significance of HRV was first recognized in 1965 when Hon and Lee [18] reported an association with fetal distress. This measurement is noninvasive and is easy to perform, so many studies were conducted using HRV. In 1996, a task force of the European Society of Cardiology and the North American Society of Pacing Electrophysiology standardized its measurement and interpretation [10]. Many previous studies utilized HRV analysis to evaluate ANS influence in various urologic disorders, including erectile dysfunction [19], chronic prostatitis [20], and female urinary incontinence [21]. Of the many HRV components, the LF component is considered to be a quantitative marker of sympathetic modulation; efferent vagal activity is a major contributor to the HF component. Thus, the LF/HF ratio is considered to be a marker that mirrors the balance of sympathetic and parasympathetic nervous activities, reflecting sympathetic modulation [10]. Although the LF/HF ratio is a good marker of ANS activity, there are no cutoff values to identify sympathetic hyperactivity. To overcome this pitfall, we performed a pilot study (unpublished data) to identify the normal value of the LF/HF ratio in elderly men without LUTS. With the approval of the institutional review board of Ajou University School of Medicine, 118 healthy volunteers from a health promotion center were included in the study and provided informed consent. They were men between the ages of 40 and 70 years, with a total IPSS lower than 8 . HRV was measured in the same manner as in this study, and a cutoff value was derived to identify sympathetic hyperactivity.

Alfuzosin is an appropriate treatment agent for patients with
$\mathrm{BPH}$ with acceptable safety profiles [22]. In this study, alfuzosin relieved LUTS regardless of sympathetic activity. Choi et al. [8] had previously performed an HRV analysis in healthy men and men with LUTS. They found that men with LUTS showed lower HF than healthy men and that patients with voiding symptom-predominant LUTS had a higher LF/HF ratio compared to patients with storage symptom predominant LUTS. We hypothesized that there would be difference in treatment outcomes according to sympathetic activity, but our study results demonstrate similar profiles of treatment efficacy. Alfuzosin is an selective a1-blocker, but it failed to show any receptor selectivity for al receptor subtypes [23,24]. This lack of subtype selectivity may be a reason to disregard differences in sympathetic activity. Other clinically available $\alpha$-blockers, such as tamsulosin and silodosin, have specific selectivity for al receptor subtypes, and their efficacy could be affected by sympathetic activity differences. Additional studies using these drugs could provide further evidence to fully describe their effects.

There are some limitations in our study. Since there is no reliable standard value to identify sympathetic hyperactivity in elderly Korean men, we performed a pilot study to identify criteria for sympathetic hyperactivity in men. Although the number of men included in the pilot study was relatively small and a comparison with LUTS patients was not performed, we think the selected criteria in this study is valuable because it was derived from a similar population as the study subjects. HRV was measured at an outpatient clinic, and there is some possibility that those hectic and crowded circumstances could influence HRV results. To exclude this possibility, we used an isolated room to measure HRV and restricted substances that could affect sympathetic activity.

Most men with symptomatic BPH could be treated with a-blockers, but there are many other patients who do not receive any benefits from the current medications. For BPH patients who have shown a poor response to the current treatments, identifying ANS dysfunction using HRV analysis could help gain a comprehensive understanding of treatment alternatives.

In conclusion, alfuzosin exhibited a good treatment effect regardless of sympathetic activity, as measured by HRV. However, patient satisfaction regarding this treatment was lower in the HSA group. Sympathetic hyperactivity may be a factor to predict treatment satisfaction, but further studies are necessary. 


\section{REFERENCES}

1. Choi SY, Kim TH, Myung SC, Moon YT, Kim KD, Kim YS, et al. Impact of changing trends in medical therapy on surgery for benign prostatic hyperplasia over two decades. Korean J Urol 2012; 53:23-8.

2. Madersbacher S, Alivizatos G, Nordling J, Sanz CR, Emberton M, de la Rosette JJ. EAU 2004 guidelines on assessment, therapy and follow-up of men with lower urinary tract symptoms suggestive of benign prostatic obstruction (BPH guidelines). Eur Urol 2004;46: 547-54.

3. Ventura S, Oliver Vl, White CW, Xie JH, Haynes JM, Exintaris B. Novel drug targets for the pharmacotherapy of benign prostatic hyperplasia (BPH). Br J Pharmacol 2011;163:891-907.

4. Roehrborn CG, Schwinn DA. Alpha1-adrenergic receptors and their inhibitors in lower urinary tract symptoms and benign prostatic hyperplasia. J Urol 2004;171:1029-35.

5. Miano R, De Nunzio C, Asimakopoulos AD, Germani S, Tubaro A. Treatment options for benign prostatic hyperplasia in older men. Med Sci Monit 2008;14:RA94-102.

6. Hammarsten J, Hogstedt B. Clinical, anthropometric, metabolic and insulin profile of men with fast annual growth rates of benign prostatic hyperplasia. Blood Press 1999;8:29-36.

7. McVary KT, Rademaker A, Lloyd GL, Gann P. Autonomic nervous system overactivity in men with lower urinary tract symptoms secondary to benign prostatic hyperplasia. J Urol 2005;174 (4Pt1): 1327-433.

8. Choi JB, Lee JG, Kim YS. Characteristics of autonomic nervous system activity in men with lower urinary tract symptoms (LUTS): analysis of heart rate variability in men with LUTS. Urology 2010; 75:138-42.

9. Low PA. Testing the autonomic nervous system. Semin Neurol 2003;23:407-21.

10. Heart rate variability: standards of measurement, physiological interpretation and clinical use. Task Force of the European Society of Cardiology and the North American Society of Pacing and Electrophysiology. Circulation 1996;93:1043-65.

11. Pleil AM, Coyne KS, Reese PR, Jumadilova Z, Rovner ES, Kelleher CJ. The validation of patient-rated global assessments of treatment benefit, satisfaction, and willingness to continue: the BSW. Value Health 2005;8 Suppl 1:S25-34.

12. Hahn DW, Lee CH, Chon KK. Korean adaptation of Spielberger's STAI (K-STAI). Korean J Health Psychol 1996;1:1-14.

13. Eckhardt MD, van Venrooij GE, Boon TA. Symptoms and quality of life versus age, prostate volume, and urodynamic parameters in 565 strictly selected men with lower urinary tract symptoms suggestive of benign prostatic hyperplasia. Urology 2001;57:695-700.

14. Shapiro E, Hartanto V, Lepor H. The response to alpha blockade in benign prostatic hyperplasia is related to the percent area density of prostate smooth muscle. Prostate 1992;21:297-307.

15. Mazur DJ, Helfand BT, McVary KT. Influences of neuroregulatory factors on the development of lower urinary tract symptoms/benign prostatic hyperplasia and erectile dysfunction in aging men. Urol Clin North Am 2012;39:77-88.

16. McVary KT, Razzaq A, Lee C, Venegas MF, Rademaker A, McKenna KE. Growth of the rat prostate gland is facilitated by the autonomic nervous system. Biol Reprod 1994;51:99-107.

17. Meigs JB, Mohr B, Barry MJ, Collins MM, McKinlay JB. Risk factors for clinical benign prostatic hyperplasia in a community-based population of healthy aging men. J Clin Epidemiol 2001;54:935-44.

18. Hon EH, Lee ST. Electronic evaluation of the fetal heart rate. VIII. Patterns preceding fetal death, further observations. Am J Obstet Gynecol 1963;87:814-26.

19. Lee JY, Joo KJ, Kim JT, Cho ST, Cho DS, Won YY, et al. Heart rate variability in men with erectile dysfunction. Int Neurourol J 2011; 15:87-91.

20. Cho DS, Choi JB, Kim YS, Joo KJ, Kim SH, Kim JC, et al. Heart rate variability in assessment of autonomic dysfunction in patients with chronic prostatitis/chronic pelvic pain syndrome. Urology 2011;78:1369-72.

21. Kim JC, Joo KJ, Kim JT, Choi JB, Cho DS, Won YY. Alteration of autonomic function in female urinary incontinence. Int Neurourol J 2010;14:232-7.

22. Roehrborn CG, Van Kerrebroeck P, Nordling J. Safety and efficacy of alfuzosin $10 \mathrm{mg}$ once-daily in the treatment of lower urinary tract symptoms and clinical benign prostatic hyperplasia: a pooled analysis of three double-blind, placebo-controlled studies. BJU Int 2003;92:257-61.

23. Kenny BA, Miller AM, Williamson IJ, O’Connell J, Chalmers DH, Naylor AM. Evaluation of the pharmacological selectivity profile of alpha 1 adrenoceptor antagonists at prostatic alpha 1 adrenoceptors: binding, functional and in vivo studies. Br J Pharmacol 1996;118:871-8.

24. Forray C, Bard JA, Wetzel JM, Chiu G, Shapiro E, Tang R, et al. The alpha 1-adrenergic receptor that mediates smooth muscle contraction in human prostate has the pharmacological properties of the cloned human alpha 1c subtype. Mol Pharmacol 1994;45:703-8. 